\title{
Face Recognition in Mobile Devices
}

\author{
Prof. Hassan \\ Soliman,Ph.D \\ Department ofcommunication \\ Engineering \\ Mansoura University \\ Egypt
}

\author{
Ahmed Saleh,Ph.D \\ Department of Computer \\ Engineering \\ Mansoura University \\ Egypt
}

\author{
Eman Fathi \\ Department of communication \\ Engineering \\ Mansoura University \\ Egypt
}

\begin{abstract}
In today's networked world,mobile phone playsvery important role, it affects all aspects of human daily life.The need to maintain the security of information in mobile device is becoming both increasingly important and increasinglydifficult. Some human features likefingerprints, face, hand geometry, voice and iris are used to provide an authentication for security systems to reach high security level instead of traditional password based systems. This paper presents a deployment of face recognition algorithms on mobile devices. Proposed approach uses PCA algorithm with FPIEand DCVon mobile device.In thispaper all calculations done on a mobile phone, in whicha small number of images were used for testing the system.System accuracy is $92 \%$ for appropriately chosen threshold, in which the time taken to recognize a face is approximately $0.35 \mathrm{sec}$ and this can increase when database size increased.
\end{abstract}

\section{Keywords}

face recognition, PCA, DCV, FPIE,mobile phones.

\section{INTRODUCTION}

In today's networked world, mobile phone playsvery important role, it affects all aspects of human daily life. The need to maintain the security of information in mobile device is becoming both increasingly important and increasinglydifficult. Most of the current phones have security for Authentication. Authentication verifies that users or systems are who they claim to be, based on identity (e.g., username) and credentials (e.g., password). Mobile devices are easily lost or stolen; moreover Password could be easily hacked or detected. For that purposehigh level of authentication for mobile devices is needed.

The term Biometrics is becoming highly important in computer security world [1].The human physical characteristics like fingerprints, face, hand,geometry, voice and iris are known as biometrics[2]. These features are used to provide an authentication for computer based security systems to reach high security level instead of traditional passwordsystems [3][4][5].Username and passwords can be replaced and/or provide double authentication by using any one of the biometric features.Face is the most suitable biometric that canbe used for authentication in mobile devices since those devices usually have cameras.
The classical face authentication process can be decomposed into several steps, namely;(i) image acquisition (grab the images from the camera in gray scale color), (ii) imageprocessing(applying filtering algorithm in order to enhance important features and to reduce the noise),(iii) face detection (detect and localize an eventual face in a given image) and finally (iv) face authentication itself, which decide if the given facecorresponds to the authenticated person or not.

The incorporation of face recognition algorithms in mobile devices becomes a challenging problem due to the constraints on processing power and limited storage of mobile devices.In the field of pattern recognition;Dimensionality reduction is an important topic of research as in many practical technologies high dimensionality is a major cause of limitation. Also the large numbers of features degrade the performance of the usedclassifiers, especially when the size of the training set is small compared to the number of features.

There is a lot of work associated with face recognition;Initial efforts to develop face recognition applications for mobile devices assumed that the mobile device only captures the image used for recognition. This image is transferred through a wireless network to a server where the actual recognition is performed, so the cost of the recognition algorithm is not a concern[6][7][. Hence the verification process depends on network availability [8][9].

Traditional face recognition works in mobile devices suffer from many hurdles, such aslarge amount of datathat are used for learning processbecause all presented algorithms leak from bad performance in small sample size. For this purpose, they used mobile deviceswith high specifications to reduce calculation time as well as consuming power.In this paper introduce a novel strategy for face recognition is introduced, which outperforms traditional techniques as it; (i) minimize recognition time, (ii)minimize consuming power during testing, (iii) maximize recognition rate. The paper is structured as follows,section 2 described basic concepts and section 3discussedprevious efforts in facerecognition areas. Section 4presented proposedmethodology by describing the features and the combination rules that used. Section 5 presented experimental results. 


\section{BACKGROUND AND BASIC CONCEPTS}

This section briefly discusses the basic principles used in this paper.Face recognitionsystem FRS,Principle Component Analysis PCA and basics of fuzzy logic systems are discussed.

\subsection{Face Recognition}

The facial recognition process normally has four interrelated phases or steps[10], which are (i) face detection, (ii) normalization, (iii) feature extraction, and (iv) face recognition. These steps depend on each other and often use similar techniques as shown in figure 1 . They may also be described as separate components of a typical FRS[11].Detecting a face in a probe image may be a relatively simple taskfor humans, but it is not so for a computer. The computer has to decide which pixels in the image is part of the face and which are not.Once the face has been detected (separated from its background), the face needs to be normalized. This means that the image must be standardized in terms of size, pose, illumination, etc., relative to the images in the gallery or reference database.After the face image has been normalized, the feature extraction and recognitionof the face can take place. In feature extraction, a mathematical representation called a biometric template or biometric reference is generated, which is stored in the database and will form the basis of any recognition task. Facial recognition algorithms differ in the way they translate or transform a face image (represented at this point as grayscale pixels) into a simplified mathematical representation (thefeatures) in order to perform the recognition task.

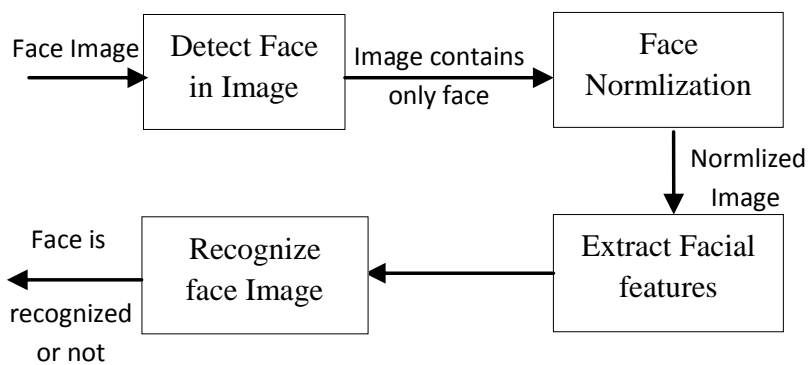

Fig1. Face Recognition Basic steps

\subsection{Principle Component Analysis -PCA}

PCA converts each two dimensional image into a one dimensional vector [12]. This vector is then decomposed into orthogonal (uncorrelated) principlecomponents (known as eigenfaces) - in other words, the technique selects the features of the image (or face) which differ from the rest of the image.Each face image is represented as a weighted sum (feature vector) of the principle components (or eigenfaces), which are stored in a one dimensional array. Each component (eigenface) represents only a certain feature of the face, which may or may not be presented in the original image. A probe image is compared against a gallery image by measuring the distance between their respective feature vectors. For PCA to work well the probe image must be similar to the gallery image in terms of size (or scale), pose, and illumination. It is generally true that PCA is reasonably sensitive to scale variation. PCA basic steps described in Figure 2.

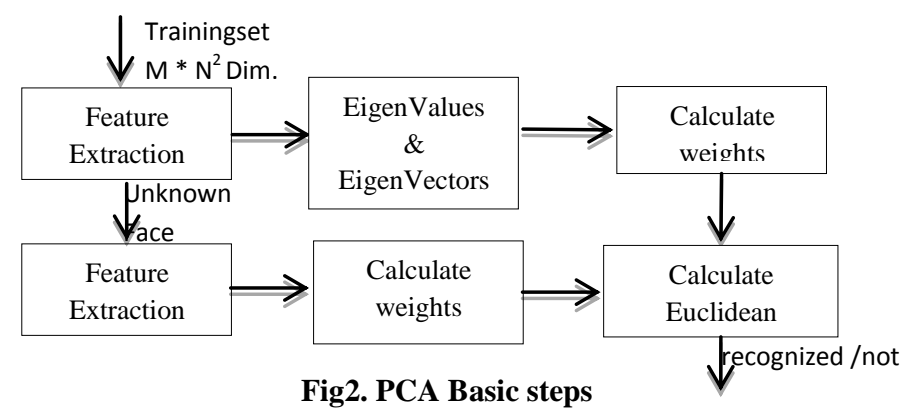

\subsection{Fuzzy logic systems}

A fuzzy logic system (FLS) can be defined as the nonlinear mapping of an input data set to a scalar output data [13][14]. Itconsists of four main parts:fuzzier, rules, inference engine, and defuzzier, which are illustrated in figure 3 . Firstly, a crisp set of input data are gathered and converted to a fuzzy set using membership functions. This step is known as fuzzification. Afterwards, an inference is made based on a set of rules (IF-THEN rules). Lastly, the resulting fuzzy output is mapped to a crisp output using the membership functions, in the defuzzification step.

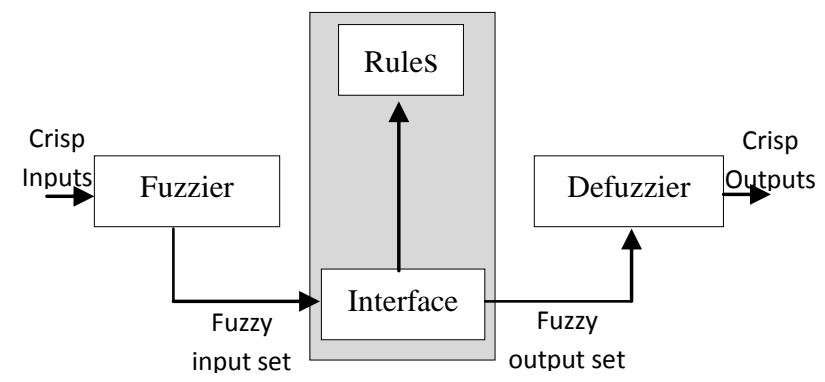

Fig3. Fuzzy logic Basic steps

\section{PREVIOUSE EFFORTS}

There are two broad of approaches for face recognition; (i) "appearance-based" approaches, in which the face image is viewed as a feature vector, (ii)"structural" approaches, in which a deformable model like a graph is used for face representation. In first kindapproach, a two-dimensional image of size $w$ by $h$ pixels is represented by a vector in a $w^{*} h$ dimensionalspace. Therefore, each facial image corresponds to a point in this space. This spaceis called the sample space or the image space, and its dimension typically is very high. Anyimage in the sample space can be represented in a lower-dimensional subspace without losing asignificant amount of information. The Eigenface also known as PCA- principle component analysis method has been proposed for finding such a lower-dimensional subspace.

The Linear Discriminant Analysis (LDA) method is proposed in [15]. This methodovercomes the limitations of the Eigenface method by applying the Fisher's Linear Discriminantcriterion. This criterion tries to maximize the ratio $\mathrm{J}_{\mathrm{FLD}}\left(\mathrm{W}_{\mathrm{opt}}\right)=\arg _{\mathrm{w}} \max \left|\mathrm{W}^{\mathrm{T}} \mathrm{S}_{\mathrm{B}} \mathrm{W}\right| /\left|\mathrm{W}^{\mathrm{T}} \mathrm{S}_{\mathrm{W}} \mathrm{W}\right|$ where $\mathrm{S}_{\mathrm{B}}$ isthe between-class scatter matrix, and $S_{\mathrm{Wis}}$ the within-class scatter matrix. In face recognition tasks, this method cannot be applied directly since the dimension of the sample space is typically larger than the number of samples in the training set. As a consequence, $S_{W}$ is singular in this case. This problem is also known as the "small sample size problem". Swets and 
Weng [16] proposed a two stage PCA+LDA method, also known asthe Fisherface method, in which PCA is first used for dimension reduction so as to make $S W$ nonsingular before the application of LDA..Jian and David [17] proposed 2DPCA method to solve the small sample size problem, 2DPCA is based on 2D image matrices rather than 1D vectors.

2 stages 2DPCA can be used to compress the image matrix redundancy, which exists among row vectors and column vectors.As proposed in [18].Another algorithm wereimplemented to solve small sample size problem , DCV Discriminative Common Vectors [19] .This approach extract the common properties of classes in the training set by eliminating the differences of the samples in each class.Virendra and Sujata in [20]brings out a new approach of information extraction based on fuzzy logic. They applied a fuzzification operation to extract the pixel wise association of face images to different classes.

In [21] a simple face recognition system based on 2D PCA is proposed, however it is only a preliminary analysis and it does not present any results. The work of Schneider [22], describes a face recognition system developedin $\mathrm{C}++$ for a Symbian platform. In [23] a face recognition system for mobile phones is presented however it requires a special camera because it is based on Near-Infrared light. The work presented in [24], Implemented on PDA's Using Viola and Jones Face detection the recognizes faces by means of correlation filters.

In [25] Color segmentation and template matching are used for Face detection then used FisherFace algorithm for Recognition. Eigen face not implemented in this work because the algorithm consumes huge power.For better performance Mauricio Villegas and Roberto Paredes in [26],they apply PCA, FisherFace and local feature algorithms on mobile devices using 8-bit(byte) integer instead of 32-byte float for computation, this leads to Memory usage reduction with PCA and Fisherface but local feature consume more time.Another research focused on finding the best threshold value used for recognition [27]. In [28] [29] face recognition in using Eigenface was implemented on HTC G1 mobile phone) and Samsung Galaxy S respectively.

This paper seeks to find the best algorithm used for mobile devices considering small memory usage and processing power. This approach used FPIE \& DCV with traditional PCA to get performance and at the same time using small samples for training.

\section{PROPOSED APPROACH}

This section presentsa new methodology for face recognition.The proposedapproach is based on small sample size for training images and small number of classes, thenit can reach best calculation time, best power consuming and at the same time reach the best performance. All images are taken after detection (In gray scale and each image only contains person's face). This approach evokes pixel wise information of face images to different classes using Fuzzy Pixel Wise Information Extraction FPIE. Training imagesare read in column vector form then apply FPIE. One fuzzy vector is generated for each face image. Discriminative common vector DCV is then appliedto find common vector corresponding to each class. By this way,number of images used for training is reduced. PCA is then applied on generated common vectors. The block diagram of implementation of proposed face recognition system is illustrated in Figure5.Each module of proposed approach will be explained in next subsections4.

\subsection{Fuzzy based Pixel wise Information Extraction (FPIE)}

FPIE module generates pixel wise degree of association of a face image to different classes using membership function (MF). This takes a face image as an input and using MF, fuzzifies the pixel values of the image. This generates the membership of individual pixel to different classes. This function can be generalized such that the values assigned to the pixels of the training set fall within a specified range which may be the unit interval $[0,1]$. Thus these values, which are real numbers in $[0,1]$, express the membership grade of the elements of the universal set. Larger values indicate higher degrees of set membership.

A face image can be represented as a $m \times n$ dimensional matrix with $m$ number of rows and $n$ number of columns. Thiscan be expressed in the form of $\mathrm{mn}$ dimensional vector $\mathrm{z}$ as:

$z=\left[z_{1} z_{2} \ldots \ldots z_{m n}\right]^{T}(1)$

a $\pi$-type MF for fuzzification [20] is used.This comprises a parameter, named fuzzifier(m), which can be tuned as per the requirement of the problem and thus provides more flexibility and generalization capability for classification.

As shown in Figure 4, the shape of this type of function is similar to that of Gaussian function. By varying the value of fuzzifier $\mathrm{m}$, the steepness of MF can be controlled. The function is given by

$$
\begin{aligned}
& \pi(\mathrm{z}, \alpha, \gamma, \beta)=0 \text {, } \\
& =2^{m-1}\left[\frac{z-\alpha}{\gamma-\alpha}\right] \quad m \\
& z \leq \alpha \\
& =1-2^{m-1}\left[\frac{\gamma-z}{\gamma-\alpha}\right] \quad m \quad c 1<z \leq \gamma \\
& =2^{m-1}\left[\frac{Z-\gamma}{\beta-\gamma}\right] \quad m \quad \gamma<z \leq c 2 \\
& =1-2^{m-1}\left[\frac{\beta-z}{\beta-\gamma}\right] \quad m \quad c 2<z \leq \beta \\
& =0 \\
& z \leq \beta
\end{aligned}
$$

$\mathrm{c} 1$ and $\mathrm{c} 2$ are crossover points

$\alpha, \gamma, \beta$ represent the minimum, maximum and mean value of training data set for a particular data point (pixel). П-type MF provides 0.5 membership grade at $\mathrm{c} 1$ and $\mathrm{c} 2$ and maximum (1.0) at the center $\gamma$ as shown in Figure 4.

the membership grade vector after applying FPIE is expressed as:

$$
g=\left[g_{1} g_{2} \ldots . g_{m n}\right]^{T}(3)
$$

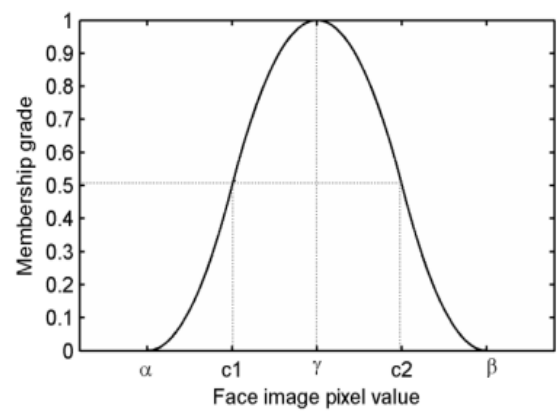

Fig4. $\pi$ membership function 
$\Phi_{i}=\varphi_{i}-\mu$

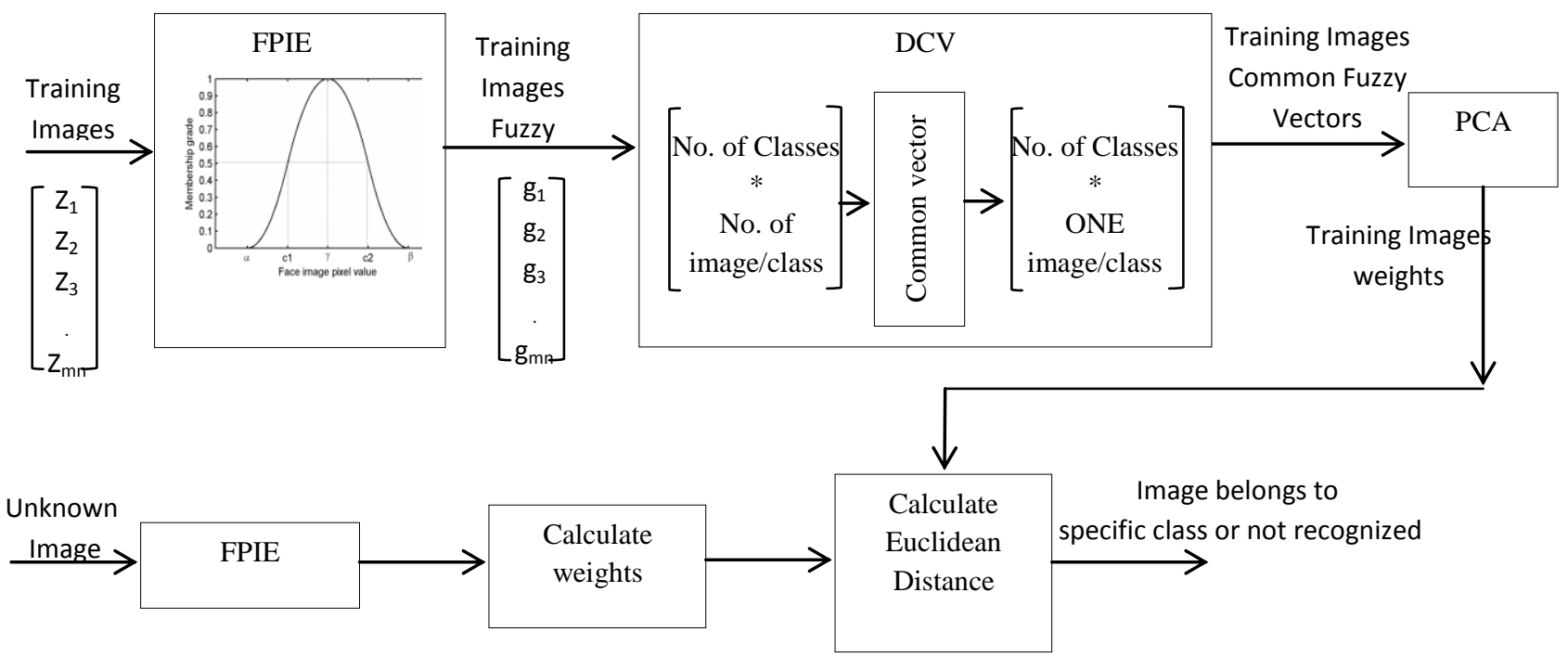

Fig5.Block Diagram of proposed architecture

\subsection{Discriminative common vector}

\section{(DCV)}

Compute the nonzero eigenvalues and corresponding eigenvectors of $S_{W}$ by using the matrix $A^{T} A$, where $S_{W} \square A A^{T}$ and $A$ is given by

$A=\left[z_{1}^{1}-\mu_{1} \ldots z_{N}^{1}-\mu_{1} z_{1}^{2}-\mu_{2} . . z_{1}^{c}-\mu_{c}\right](4)$

Where $\mathrm{z}$ is defined as (1) and $\mathrm{c}$ is the number of classes used. Set $Q \square \square \notin 1 \ldots \alpha r$, where $r$ is the rank of $S_{W}$,where $Q$ is the eigenvectors corresponding to nonzero eigenvalues of $\mathrm{S}_{\mathrm{w}}$. Choose any sample from each class and project it onto the null space of $S_{W}$ to obtain thecommon vectors

$Z_{c o m}^{i}=z_{m}^{i}-Q(5)$

After obtaining the common vector $\mathrm{Z}_{\text {com }}^{\mathrm{i}}$, construct $\mathrm{S}_{\text {com }}$ matrix where

$$
S_{\text {com }}=\sum_{i=1}^{c}\left(Z_{\text {com }}^{i}-\mu_{\text {com }}\right)\left(Z_{c o m}^{i}-\mu_{\text {com }}\right)^{T}
$$

Where $\mu_{\mathrm{com}}$ is the mean of all common vectors .

$\mu_{\text {com }}=\frac{1}{c} \sum_{i=1}^{c} Z_{\text {com }}^{i}$

\subsection{Principle Component Analysis (PCA)}

The eigenface scheme is pursued as a dimensionality reduction approach, more generally known as principal component analysis (PCA), or Karhunen-Loeve method. Such method chooses a dimensionality reducing linear projection that maximizes the scatter of all projected images. Given a training set of $\mathrm{N}$ images $\Gamma i(i=1,2, \ldots \mathrm{N})$, each of size $\mathrm{m} \times \mathrm{n}$, it could be turned into a big matrix as

$A=\left[\begin{array}{lll}\Phi_{1} & \Phi_{2} & \Phi_{N}\end{array}\right]$

where $\Phi$ i's are column vectors, each corresponding to an image. Average face is calculated as $\mu=\operatorname{mean} \varphi_{i}$

The total scatter matrix is defined as

$S_{T}=A A^{T}$

PCA leads to find the projection $\mathrm{W}_{\text {opt }}$ that maximizes the determinant of the total scatter matrix of the projected images.

$W_{\text {opt }}=\arg _{w} \max \left|W^{T} S_{T} W\right|$

Where wi's are eigenvectors of ST corresponding to the $p$ largest eigenvalues. Each of them corresponds to an "eigenface". The dimension of the feature space is thusreduced to $\mathrm{p}$. The weights of the training set images and test images could be then calculated and the Euclidean distances are obtained. The test face is recognized as the face of training set with the closest distance, if such distance is below a certain distance as shown in Figure 5.

In this approach, number of samples used for training is reduced, to reduce the timeof recognition formobile environment and atthe same time get the best performance. Using FPIE, then find the common vector that represents each class using DCV, to reduce number of samples again. Finally PCA is added for testing .This approach outperforms other traditional techniques as shown in results.

\section{EXPERIMENTAL RESULTS}

For the experiments, conventional computer is used as mobile device. Computer includes $2 \mathrm{GHz}$ CPU, 2 GB memory. Images used were in gray scale and contain only faces as shown in Figure 8, First group of images used withoutfacial expression or pose variation, second images used from AT\&T face database [30] with facial expression and pose variation, as shown in figure 8. Parameters used for testing performance are time (milliseconds) and accuracy (recognized Images/Total Number of tested image). All experiments done on testing set contain 25 images, Images contain persons belong to trainingset and did not belong to training set. 


\subsection{Competitors}

Proposed approach comparedwith face recognition algorithms listed in Table1. All results are taken in thesame environment and same training set.

\section{Table 1. Competitors Description}

\begin{tabular}{|c|l|}
\hline Method & \multicolumn{1}{c|}{ Description } \\
\hline $\begin{array}{c}\text { PCA - Principle } \\
\text { Component } \\
\text { Analysis }\end{array}$ & $\begin{array}{l}\text { Dimensionality reduction approach } \\
\text { used for linear projection that } \\
\text { maximizes the scatter of all projected } \\
\text { images, image converted to one } \\
\text { dimensional vector. }\end{array}$ \\
\hline $\begin{array}{c}\text { 2DPCA - } \\
\text { Dimensional } \\
\text { PCA }\end{array}$ & $\begin{array}{l}\text { PCA Enhancement to solve small } \\
\text { sample size problem by using each } \\
\text { image as 2 dimension matrix. }\end{array}$ \\
\hline 2Satge 2DPCA & $\begin{array}{l}\text { 2DPCA enhancement that reduce } \\
\text { dimensionality in both columns and } \\
\text { rows for image matrix. }\end{array}$ \\
\hline $\begin{array}{c}\text { FPIE Fuzzy } \\
\text { Pixel wise } \\
\text { Information } \\
\text { Extraction }\end{array}$ & $\begin{array}{l}\text { Generates pixel wise degree of } \\
\text { association of a face image to different } \\
\text { classes using membership function } \\
\text { (MF) then Apply PCA on results. }\end{array}$ \\
\hline
\end{tabular}

\subsection{First Experiment}

In this experiment $25 * 21$ pixels image resolution is used, images were selected without facial or pose variations. Training set contains 3 classes ( 3 persons) each class contains 3 images.PCA takes $200 \mathrm{~ms}$ for testing and indicates 30\% recognition accuracy, that's for PCA doesn't perform well for small size of training set. 2DPCA takes $265 \mathrm{~ms}$ and has 85\% accuracy, 2DPCA indicates more accuracy but it consumes more power for 2D matrix calculation. 2-Stage 2DPCA takes $250 \mathrm{~ms}$ and has $92 \%$ accuracy but in this case testing time is reduced. FPIE indicates $190 \mathrm{~ms}$ and $30 \%$ accuracy.

In proposed approach,Learning time was around $353 \mathrm{~ms}$, testing time was 200 msand recognition accuracy around $92 \%$. Experiment proved that proposed approach outperformed other approaches when small number of training set are used, it gave better accuracy and consume less power as shown in figure 6 .

\subsection{Second Experiments}

In this experiment50*61 pixels image resolution is used, images were selectedwith facial and pose variations. Training set contains 3 classes each contains 5 images;same experiment is repeated for 7 and 9 images/class. In this experiment training images used contained pose and facial expressions variation.

\subsubsection{Using 5 images per class}

PCA gave $33 \%$ accuracy, $330 \mathrm{~ms}$ testing time , FPIE gave $20 \%$ accuracy, $290 \mathrm{~ms}$ testing time . Accuracy for 2DPCA and 2-2DPCA gave $87 \%$ accuracy, testing time was $440 \mathrm{~ms}$ for 2DPCA and $400 \mathrm{~ms}$ for 2-2DPCA . proposed approach gave $80 \%$ accuracy and $325 \mathrm{~ms}$ testing time.

\subsubsection{Using 7 images per class}

PCA gave $37 \%$ accuracy, $345 \mathrm{~ms}$ testing time. FPIE gave $20 \%$ accuracy , $300 \mathrm{~ms}$ testing time . Accuracy for 2DPCA and 2-2DPCA gave $90 \%$ accuracy, testing time was $530 \mathrm{~ms}$ for 2DPCA and $510 \mathrm{~ms}$ for 2-2DPCA . proposed approach gave $88 \%$ accuracy and $350 \mathrm{~ms}$ testing time.

\subsubsection{Using 9 images per class}

PCA gave $40 \%$ accuracy, $370 \mathrm{~ms}$ testing time . FPIE gave $20 \%$ accuracy , $320 \mathrm{~ms}$ testing time . Accuracy for 2DPCA and 2-2DPCA gave $96 \%$ accuracy, testing time was $570 \mathrm{~ms}$ for 2DPCA and $550 \mathrm{~ms}$ for 2-2DPCA . proposed approach gave $95 \%$ accuracy and $370 \mathrm{~ms}$ testing time.

As shown in figure 6, experiments showed that(i) 5 images/class experiment accuracy reduced for all algorithms because of facial and pose variations, then accuracy increased as number of images per class increased. (ii) The worthiest cases were PCA \& FPIE, that because they didn't perform well with small number of training samples, 2DPCA and 22DPCA gave more accuracy but they took more testing time (more consuming power). (iii) Proposed approach outperforms other algorithms in testing time and recognition accuracy,it gives the same good accuracy as 2DPCA and 22DPCA but with small testing time.

As shown in Figure 7, experiments proved that, (i) proposed approach overcome small sample size problem when the dimension of the sample space is larger than the number of training set,because this case started with 3 images per class and experiment give good result, (ii) testing time is small which will be suitable for mobile devices because it will be faster than other algorithms, (iii) recognition accuracy is high compared to other approaches. 

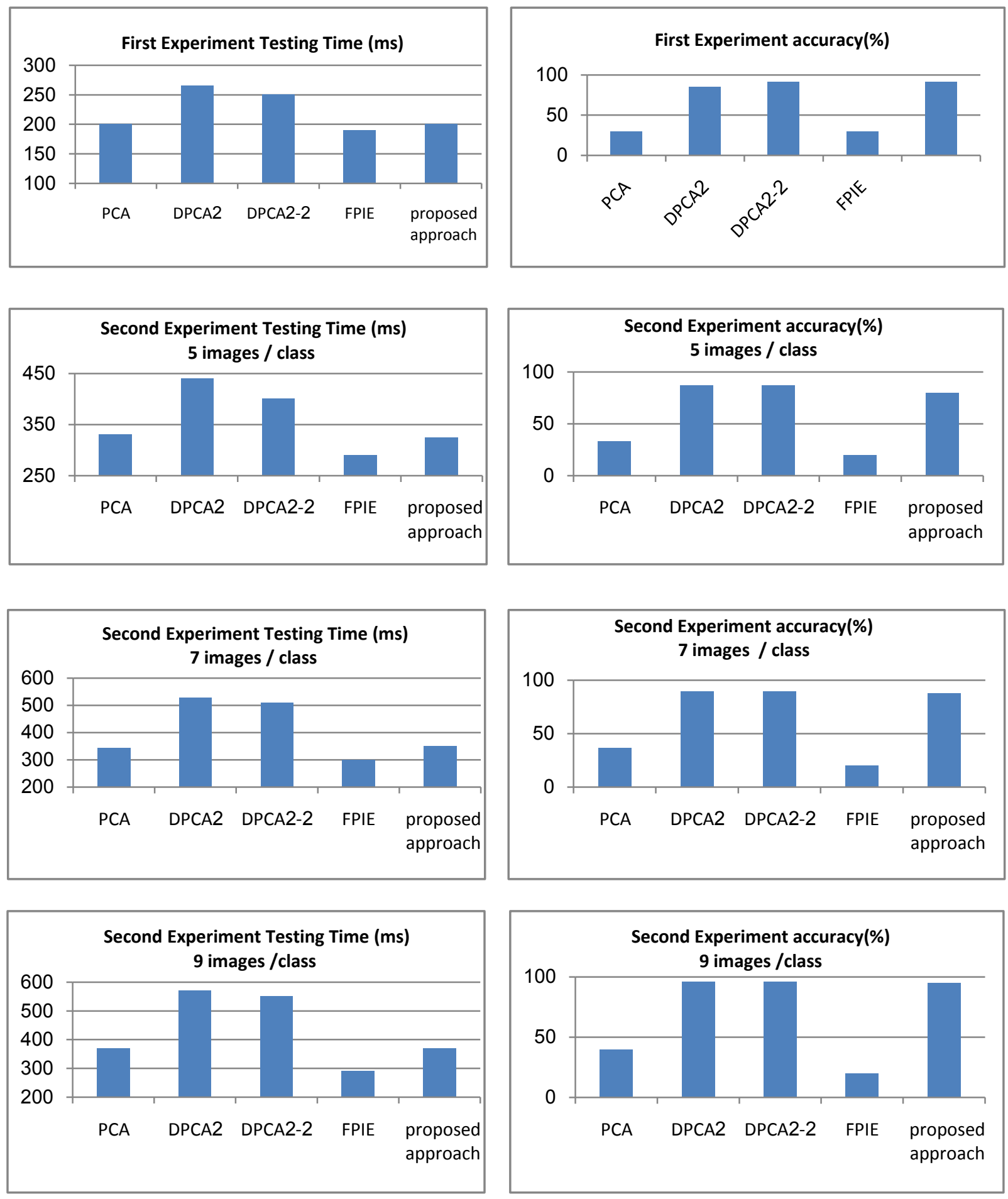

Fig6. Experimental results graph 

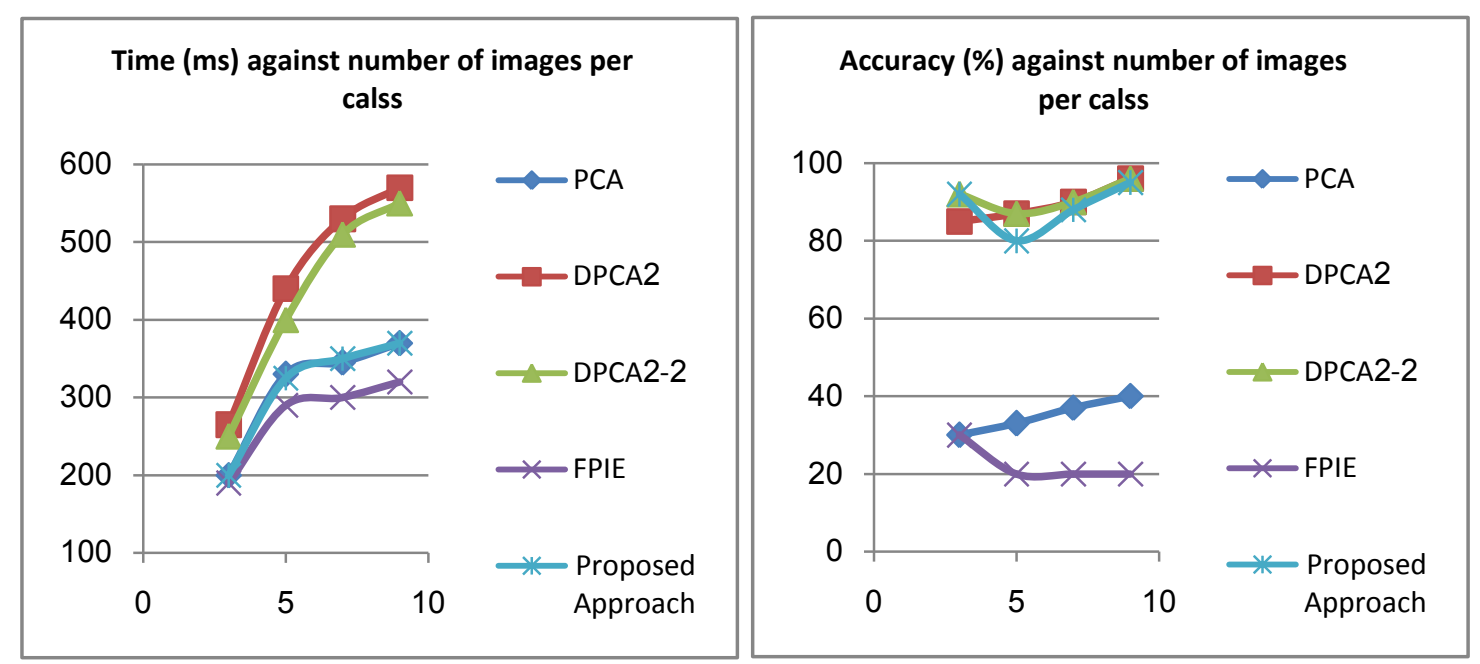

Fig7. Time and Accuracy comparison for Second Experiment

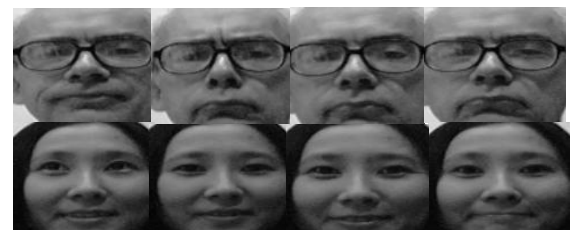

(a)

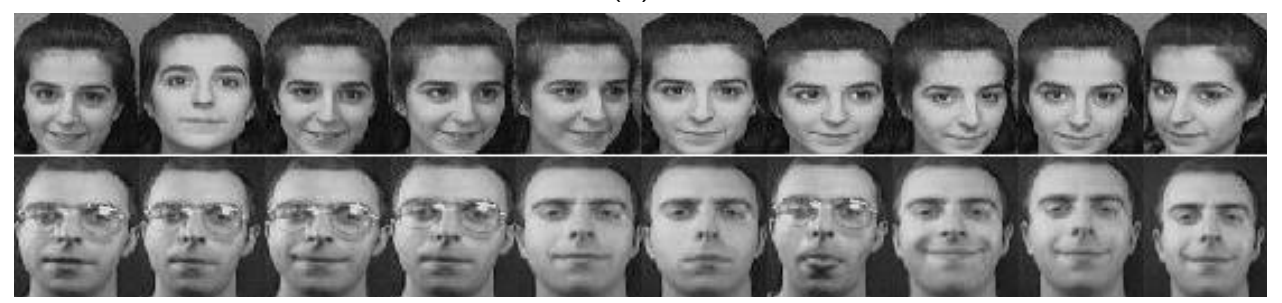

(b)

Fig8. Sample images used for training: (a) First group, (b) AT\&T 


\section{CONCLUSION}

Face recognition tasks has been proposed. This approach unifies the capability of fuzzy set theory to obtain the degree of belonging of different pixels of a face image to different classes. Common vector method is obtained to reduce the number of samples used in training then traditional PCA has been used for recognition task. Thisshows the significant improvement in classification accuracyand recognition time. Future work will enhance this approach by detectingoutlier's pixels to exclude distorted images from learning process to get better recognition accuracy and performance.

\section{REFRENCES}

[1] Joseph Lewis, University of Maryland, Bowie State University,January 2002,Biometrics for secure Identity Verification: Trends and Developments.

[2] Biometric Recognition2003 ,Security and Privacy Concerns.

[3] Kresimir Delac , Mislav Grgic 2004 ,a survey of biometric recognition methods.

[4] sulochana sonkamble, 2dr. ravindra thool, 3balwant sonkamble 2010, survey of biometric recognition systems and their applications, journal of theoretical and applied information technology.

[5] Anil $\mathrm{K}$.

Jain, Ajay2010Kumar,"Biometrics of Next Generation: An Ov erview .

[6] Eugene Weinstein, Purdy Ho, Bernd Heisele,Tomaso Poggio, Ken Steele, and Anant Agarwal 2002,Handheld Face Identification Technology in a Pervasive Computing Environment.

[7] Etisalat Coll. of Eng., Sharjah,United Arab Emirates 2005, A GPRS-based remote human face identification system for handheld devices.

[8] Timothy J. Hazen, Eugene Weinstein and Alex Park 2003, Towards Robust Person Recognition On Handheld Devices Using Face and Speaker Identification Technologies.

[9] Shibnath Mukherjee,Zhiyuan Chen 2006, A Secure Face Recognition System for Mobile-devices without The Need of Decryption.

[10] Shang-Hung Lin, Ph.D. 2002,An Introduction to Face Recognition Technology.

[11] Wei-Lun Chao 2010, Face Recognition.

[12] M. Turk, and A. Pentland, Eigenfaces for Face Recognition, Journal of Cognitive Neuroscience, vol. 3 num.1 (1991) 71-86.

[13] Laboratoire, Equipe 2000 ,Fuzzy Logic Introduction.

[14] J. Mendel.1995 Fuzzy logic systems for engineering: a tutorial. Proceedings of the IEEE, 83(3):345\{377.

[15] Juwei Lu, K.N. Plataniotis, and A.N. Venetsanopoulos,"Face Recognition Using LDA Based Algorithms", May 2002.
[16]D. L. Swets and J. Weng 1996, Using discriminant eigenfeatures for image retrieval, IEEE Transaction on Pattern Analysis and Machine Intelligence, vol. 18, no. 8, pp. 831-836.

[17] Jian Yang, David Zhang 2004, Two-Dimensional PCA: A New Approach to Appearance-Based Face Representation and Recognition ,IEEE Transaction on Pattern Analysis and Machine Intelligence, vol. 26, no. 1.

[18] Dong Xu1, Shuicheng Yan2, Lei Zhang, Mingjing Li, Weiying Ma, Zhengkai Liu1, Hongjiang Zhang,Parallel Image Matrix Compression for Face Recognition

[19] Hakan Cevikalp, Student Member2005, IEEE, Marian Neamtu,Discriminative Common Vectors for Face Recognition, IEEE Transaction on Pattern Analysis and Machine Intelligence, vol. 27, no. 1.

[20] Virendra P. Vishwakarma, Member2010, IACSIT, Sujata Pandey, Member, IEEE and M. N. Gupta, Fuzzy based Pixel wise Information Extraction for Face Recognition IACSIT International Journal of Engineering and Technology vol. 2, no.1.

[21] Daijin Kim Sang-Ho Cho, Bong-Jin Jun2005. Face recognition on a mobile device. In Proceedings of International workshop of Intelligent Information Processing .

[22] C. Schneider, N. Esau, L. Kleinjohann, and B. Kleinjohann 2006. Feature based face localization and recognition on mobile devices. Control, Automation, Robotics and Vision,. ICARCV '06. 9th International Conference on, pages 1 .

[23] Song yi Han, Hyun-Ae Park, Dal Ho Cho, Kang Ryoung Park, and Sangyoun Lee2007. Face recognition based on near-infrared light using mobile phone. In ICANNGA (2), pages $440-448$.

[24] Chee Kiat Ng, Marios Savvides, and Pradeep K. Khosla.2005 Real-time face verification system on a cellphone using advanced correlation filters. Automatic Identification Advanced Technologies, IEEE Workshop on, 0:57-62.

[25] Guillaume Dave, Xing Chao, Kishore Sriadibhatla, " Face Recognition in Mobile phones by" 2003.

[26] Mauricio Villegas , Roberto Paredes2010, On Optimising Local Feature Face Recognition for Mobile Devices by V Jornadas de Reconocimiento Biom'etrico de Personas.

[27] Sheifali Gupta , O.P.Sahoo , Ajay Goel, Rupesh Gupta ,A2010 New Optimized Approach to Face Recognition Using EigenFaces.

[28] Charalampos Doukas and Ilias Maglogiannis 2010,A Fast Mobile Face Recognition System for Android OS Based on Eigenfaces Decomposition

[29] Emir Kremić, Abdulhamit Subaşi2011 ,The Implementation of Face Security for Authentication Implemented on Mobile Phone.

[30] AT\&T face database, [Online]. Available: http://www.cl.cam.ac.uk/Research/DTG/attarchive/pub/d ata/att faces.t ar.Z 